\title{
Counterterm method and thermodynamics of hairy black holes in a vector-tensor theory with Abelian gauge symmetry breaking
}

\author{
Ai-chen $\mathrm{Li} \odot^{*}$ \\ Departamento de matematica da Universidade de Aveiro and CIDMA, \\ Campus de Santiago, 3810-183 Aveiro, Portugal and Institute of Theoretical Physics, \\ Beijing University of Technology, Beijing 100022, China
}

(Received 12 June 2021; accepted 16 July 2021; published 16 August 2021)

\begin{abstract}
For a type of nonminimally coupled vector-tensor theories with Abelian gauge symmetry breaking in four-dimensional spacetime and correspondingly asymptotic non-AdS black hole solutions including a cosmological constant, we construct the appropriate boundary terms and derive the associated junction condition. In order to remove the divergences in the stress tensor which is localized on the spacetime boundary, we also involve the suitable surface counterterms into the total action. Using the counterterm method, we calculate the black hole mass. An implicit relation between the black hole carge $Q$ and other parameters is implied by combining the expression of the black hole mass with the first law of black hole thermodynamics. With this implicit relation, we can prove the inequality $Q \leq M$ which is a general bound for most of charged black holes. Besides, the phase structure of black holes is also investigated in the grand canonical ensemble.
\end{abstract}

DOI: 10.1103/PhysRevD.104.044040

\section{INTRODUCTION}

In recent decades, the studies about hairy black holes have become a hot topic. One motivation is the anti-de Sitter space/conformal field theory correspondence [1-3], i.e., AdS/CFT. This correspondence suggests that the physics of strongly coupled gauge field living on boundary of AdS spacetime could be reproduced by classical or semiclassical gravitational theory in the bulk. In application of AdS/CFT, the hairy black holes are used to understand quantum chromodynamics (QCD) at finite temperature [4-6], superconductor's behavior $[7,8]$, and other interesting phase transitions [9-14]. Besides, another motivation comes from the reexamination to the black hole no-hair theorems. According to them, in four-dimensional spacetime with gravity, black holes are characterized only by three physical parameters, namely the mass, electric charge, and angular momentum. And the existence of black holes characterized by other parameters are excluded. However, some interesting counterexamples have been constructed in recent years see e.g., [15-27]. Moreover, recently the phenomenon of black hole spontaneous scalarization has been the focus of considerable attention-see, e.g., [28-36]. Specifically,

\footnotetext{
*lac@emails.bjut.edu.cn
}

Published by the American Physical Society under the terms of the Creative Commons Attribution 4.0 International license. Further distribution of this work must maintain attribution to the author(s) and the published article's title, journal citation, and DOI. Funded by SCOAP ${ }^{3}$. these models consider a nonminimal coupling between the scalar field $\phi$ and some source therm $I$, which could produce a repulsive gravitational effect. In this way, the black hole solution with no hair, from Einstein's gravity, is unstable against scalar perturbations due to the source, and the scalar (or more general vector or tensor) hair will grow dynamically during this process.

Most hairy black hole solutions have been considered in scalar-tensor theories or scalar-vector-tensor theories. Solutions from vector-tensor theories have been less investigated. Actually, many interesting cosmological phenomenology have been found in vector-tensor theories, especially for those with Abelian symmetry breaking [37-47]. Recently, a class of interesting black hole solutions in four-dimensional spacetime were obtained by [48] from a type of vector-tensor theory in which the Abelian gauge symmetry is broken by coupling the vector with gravity in a nonminimal way $[49,50]$.

In this paper, our purpose is to find the appropriate boundary terms and construct the effective holographic surface counterterms for a type of nonminimally coupled vector-tensor theory and correspondingly asymptotic nonAdS black hole solutions including a cosmological constant [48]. After obtaining the black hole mass via a surface counterterms method, we also intend to investigate the relevant thermodynamics. Our motivation comes from the following aspects, both in physics and in techniques. As we know, for the Reissner-Nordström black hole, the value of the charge $Q$ should be less than the value of the black hole mass $M$; otherwise no event horizon exists. More generally 
speaking, the extremality bound $Q \leq M$ holds for most of charged black hole solutions, of course, there also exists some interesting counterexamples [51,52]). However, for black hole solutions including a cosmological constant in [48], the value of $Q$ is arbitrary and has no effect on the existence of horizon. It seems that there is no direct evidence to show $Q \leq M$ or $Q>M$ or both are allowed. Thus, after the consideration of thermodynamics for these black hole solutions, we expect to find a constraint relation between $Q$ and other physical parameters. In this way, we can compare $Q$ and $M$ at other fixed physical parameters. Furthermore, the thermodynamics for black hole solutions derived from some typical vector-tensor theories with broken Abelian gauge symmetry has been investigated by works $[53,54]$, via the Wald formalism. These works derive the general first laws of black hole thermodynamics in either minimally or nonminimally coupled case. But the physics behind these formulas have not been thoroughly investigated. In this work, we expect to gain some intuition about the black holes in vector-tensor theory without Abelian gauge symmetry [48] by analyzing the phase structures in the grand canonical ensemble.

From the viewpoint of techniques, due to the presence of a nonminimal coupling between the vector field and gravity in the action given in [48], the Gibbons-Hawking term [55] is not the appropriate boundary term here. The effective boundary term needs to be obtained. Besides, for obtaining a finite quasilocal stress tensor on spacetime boundary, some suitable surface counterterms should be added to the total action. This process is the so called holographic renormalization [56-62]. However, in [48], there exists nonminimal coupling between vector field and gravity, while the black hole solution is not an exactly asymptotic AdS geometry. Thus, the surface counterterms will be more complicated than [60]. At present, there are few research works about resolving these technical problems. Thus, to enrich the studies about the vector-tensor theories with Abelian symmetry breaking, we shall consider these technical problems for [48] in this work.

Our work is structured as follows. In Sec. II, we briefly review a type of vector-tensor theory with broken Abelian gauge symmetry in four-dimensional spacetime and correspondingly asymptotic non-AdS black hole solutions including a cosmological constant. In Sec. III, we construct the appropriate boundary terms and derive the corresponding generalized Israel junction conditions on the spacetime boundary. Besides, we also give a welldefined quasilocal stress tensor and calculate the black hole mass by adding the suitable surface counterterms to the total action. After obtaining the black hole mass, the relevant thermodynamics, including the first law of black hole thermodynamics and the phase structure analysis in the grand canonical ensemble, have been considered in Sec. IV. Finally, we will summarize our results and give a discussion in Sec. V.

\section{BLACK HOLES SOLUTION INCLUDING A COSMOLOGICAL CONSTANT IN VECTOR-TENSOR THEORY WITH ABELIAN SYMMETRY BREAKING}

An analytical black hole solution in 4-dimensional spacetime has been found in a type of vector-tensor theory [48]. The action is set as

$S=\frac{1}{2 \kappa^{2}} \int \sqrt{-g} d^{4} x\left\{R-2 \Lambda-\frac{1}{4} F^{2}+\beta G_{\mu \nu} A^{\mu} A^{\nu}\right\}$

in which the $G_{\mu \nu}$ is the standard Einstein tensor, while the $\beta$ is the physical constant which measures the strength of nonminimal coupling between the vector field and Einstein tensor. It is easy to see that the $U(1)$ symmetry is broken in presence of this nonminimal coupling term. The Einstein field equation is given by

$$
\begin{aligned}
R_{\mu \nu}-\frac{1}{2} g_{\mu \nu} R= & -\Lambda g_{\mu \nu}+\beta Z_{\mu \nu}+\frac{1}{2}\left(F_{\mu \sigma} F_{\nu}^{\sigma}-\frac{1}{4} g_{\mu \nu} F^{2}\right) \\
Z_{\mu \nu}= & \frac{1}{2} A^{2} R_{\mu \nu}+\frac{1}{2} R A_{\mu} A_{\nu}-2 A^{\alpha} R_{\alpha(\mu} A_{\nu)}-\frac{1}{2} \nabla_{\mu} \nabla_{\nu} A^{2} \\
& +\nabla_{\alpha} \nabla_{(\mu}\left(A_{\nu} A^{\alpha}\right)-\frac{1}{2} \nabla^{\alpha} \nabla_{\alpha}\left(A_{\mu} A_{\nu}\right) \\
& +\frac{1}{2} g_{\mu \nu}\left(G_{\alpha \beta} A^{\alpha} A^{\beta}+\nabla^{\alpha} \nabla_{\alpha} A^{2}-\nabla_{\alpha} \nabla_{\beta}\left(A^{\alpha} A^{\beta}\right)\right)
\end{aligned}
$$

the equation of motion for the vector field, namely the extended Maxwell equations, reads

$$
\nabla_{\mu} F^{\mu \nu}+2 \beta A_{\mu} G^{\mu \nu}=0
$$

The metric ansatz is set up as

$$
d s^{2}=-h(r) d t^{2}+\frac{d r^{2}}{f(r)}+r^{2}\left(d \theta^{2}+\sin ^{2} \theta d \phi^{2}\right)
$$

Meanwhile, $A_{\mu}$ has the following ansatz

$$
A_{\mu} d x^{\mu}=a(r) d t+\chi(r) d r
$$

After substituting ansatz (4) and (5) into Einstein field equations (2) and extend Maxwell equations (3), the following four independent differential equations are given [48]

$$
\frac{f^{2}}{r}-\frac{f}{r}+\frac{h^{\prime} f^{2}}{h}=0
$$

$$
\begin{array}{r}
4 \beta a\left(1-f-r f^{\prime}\right)+a^{\prime}\left(r-5 r f-r^{2} f^{\prime}\right)-2 r^{2} a^{\prime \prime} f=0 \\
4 \beta\left(a^{2}(f-1)+f\left(h \chi^{2}+2 r a a^{\prime}\right)\right)+r^{2}\left(4 \Lambda h+a^{\prime 2} f\right)=0
\end{array}
$$




$$
\begin{aligned}
& 2 \beta\left(a^{2}(1-f)+\chi^{2} f h(1+f)\right)+4 h\left(f+r^{2} \Lambda-1\right) \\
& +r^{2} f\left(a^{\prime 2}+\frac{4 h f^{\prime}}{r f}\right)+2 r \beta f^{2} \chi^{2}\left(\frac{3 h f^{\prime}}{f}-\frac{a^{2} f^{\prime}}{\chi^{2} f^{2}}+\frac{4 h \chi^{\prime}}{\chi}\right)=0
\end{aligned}
$$

When $\beta=\frac{1}{4}$, an analytical solution of spherically symmetric black hole is given as

$$
\begin{array}{r}
h(r)=1-\frac{2 m}{r}+\frac{4 r^{2} \Lambda_{\mathrm{eff}}}{3}+\frac{4}{5} r^{4} \Lambda_{\mathrm{eff}}^{2} \\
f(r)=\left(1+2 r^{2} \Lambda_{\mathrm{eff}}\right)^{-2} h(r) \\
a(r)=\frac{Q}{r}+Q_{2}\left(1+\frac{2}{3} r^{2} \Lambda_{\mathrm{eff}}\right) \\
\chi(r)=\frac{1+2 r^{2} \Lambda_{\mathrm{eff}}}{\sqrt{h}}\left(\frac{\left(3 Q+r Q_{2}\left(3+2 r^{2} \Lambda_{\mathrm{eff}}\right)\right)^{2}}{9 r^{2} h}\right. \\
\left.-Q_{2}^{2}\left(1+2 r^{2} \Lambda_{\mathrm{eff}}\right)+16 r^{2} \Lambda_{\mathrm{eff}}\right)^{\frac{1}{2}}
\end{array}
$$

where

$$
\Lambda_{\mathrm{eff}}=\frac{2 \Lambda}{Q_{2}^{2}-8}
$$

From (10), we see that the effective cosmological constant in the black hole solutions is (14). Thus, for obtaining an effective negative cosmological constant, we will only consider the case $Q_{2}^{2}>8$ in Sec. III and Sec. IV. Actually, one could also consider the case $\Lambda>0 \& Q_{2}^{2}<8$ as an alternative way. And the same results will be observed. Besides, note that the asymptotic behavior of black hole solutions at large $r$ is

$d s^{2} \sim-\frac{4 \Lambda_{\mathrm{eff}}^{2}}{5} r^{4} d t^{2}+\frac{1}{5} d r^{2}+r^{2}\left(d \theta^{2}+\sin ^{2} \theta d \phi^{2}\right)$.

It is easy to see that this black hole solutions including a cosmological constant in [48] are not asymptotic AdS geometry.

We plot $h(r)$ in Fig. 1 at some fixed physical parameters $\Lambda, m$ with varying $Q_{2}$. It is easy to see that there only exists

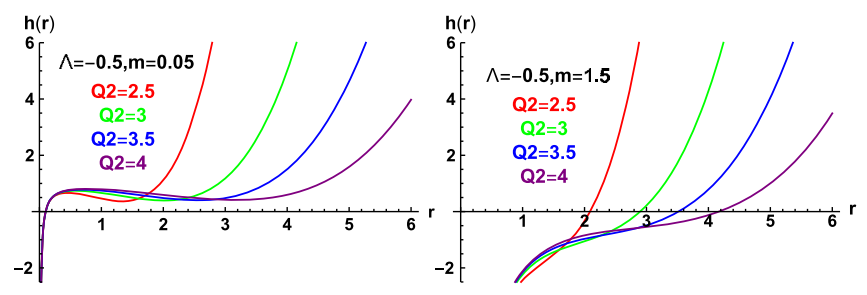

FIG. 1. Plot the horizon function $h(r)$ (10) at fixed $\Lambda, m$ with different $Q_{2}$. one horizon $r_{h}$ for this black hole solution whatever the value of $\Lambda, m, Q_{2}$. Besides, from (10)-(13), it is not difficult to observe that the value of $Q$ is arbitrary with respect to other parameters and has no effect on the existence of a horizon $r_{h}$.

\section{JUNCTION CONDITION, COUNTERTERM METHOD AND HOLOGRAPHIC ENERGY}

In this part, we need to construct some physical quantities on the spacetime boundary $r=\infty$. Before that, some notation should be introduced. We use $x^{\mu}=$ $(t, r, \theta, \phi)$ and $d s^{2}=g_{\mu \nu} d x^{\mu} d x^{\nu}$ to denote the coordinates and metric in the bulk spacetime. In the spacetime boundary, namely $r=\infty$, the coordinates are denoted as $x^{a}=(t, \theta, \phi)$. Thus, the 4-velocity of the boundary hypersurface is easily obtained as $u^{\mu}=(1,0,0,0)$, and the unit normal pointing into the boundary hypersurface is $n_{\nu}=\left(0, \frac{1}{\sqrt{f}}, 0,0\right)$. Then the induced metric of the boundary spacetime could be obtained as

$d s^{2}=\gamma_{a b} d x^{a} d x^{b}=-h(r) d t^{2}+r^{2}\left(d \theta^{2}+\sin ^{2} \theta d \phi^{2}\right)$

$\gamma_{a b}=e_{a}^{\mu} e_{b}^{\nu} \gamma_{\mu \nu}$

where the vielbein $e_{a}^{\mu}$ is defined as $e_{a}^{\mu}=\frac{\partial x^{\mu}}{\partial x^{a}}$, which is tangent to the boundary and satisfies $n_{\mu} e_{a}^{\mu}=0$. Besides, the projection tensor $\gamma_{\mu \nu}$ is defined as $\gamma_{\mu \nu}=g_{\mu \nu}-n_{\mu} n_{\nu}$, whose tangential components $\gamma_{a b}$ correspond to the induced metric on spacetime the boundary. For calculating the quasilocal stress tensor on spacetime the boundary, we need to add the boundary terms to the action (1). As it is known, when varying the Einstein-Hilbert action with respect to the metric tensor $g_{\mu \nu}$, besides a bulk term which yields the standard Einstein field equation, we also obtain the following boundary term

$$
\frac{1}{2 \kappa^{2}} \int \sqrt{-\gamma} d^{3} x\left\{g_{\alpha \beta} n_{\rho} \nabla^{\rho}\left(\delta g^{\alpha \beta}\right)-n_{\rho} \nabla_{\lambda}\left(\delta g^{\rho \lambda}\right)\right\}
$$

in which the $n_{\rho}$ is the unit normal vector pointing into the spacetime boundary. Note that in expression of (17), the derivative of the metric variation in the normal direction is discontinuous across the boundary hypersurface. For cancelling the $\nabla^{\rho}\left(\delta g^{\alpha \beta}\right)$-like terms and making the metric variation well defined on the spacetime boundary, a Gibbons-Hawking boundary term [55] is involved

$$
S_{\mathrm{GH}}=\frac{1}{\kappa^{2}} \int d^{3} x \sqrt{-\gamma} K
$$

where $K=\gamma^{\mu \nu} K_{\mu \nu}$ is the trace of the extrinsic curvature tensor $K_{\mu \nu}=\frac{1}{2}\left(\nabla_{\mu} n_{\nu}+\nabla_{\nu} n_{\mu}\right)$. Adding the (17) with the variation of the Gibbons-Hawking term together, we will obtain the following Israel junction term [63-65] 


$$
\frac{1}{\kappa^{2}} \int \sqrt{-\gamma} d^{3} x\left\{D^{\nu}\left(\gamma_{\nu}^{\alpha} n^{\beta} \delta g_{\alpha \beta}\right)+\left(K_{\mu \nu}-h_{\mu \nu} K\right) \delta g^{\mu \nu}\right\}
$$

in which $D^{\nu}$ is the covariant differentiation with respect to $\gamma_{\mu \nu}$. Thus, the first term in (19) is a total derivative and could be thrown away. In the case of Einstein-Proca theory (1), besides the Einstein-Hilbert action, the nonminimal coupling $\frac{1}{2 \kappa^{2}} \int \sqrt{-g} d^{4} x \beta G_{\mu \nu} A^{\mu} A^{\nu}$ will also lead to a pathological boundary term when calculating the variation with respect to $g^{\mu \nu}$. Thus we also need to introduce an appropriate boundary term to make the nonminimal coupling term have a well-defined behavior on the spacetime boundary.

\section{A. An appropriate boundary term which corresponds to the nonminimal coupling terms}

Varying the term $\frac{1}{2 \kappa^{2}} \int \sqrt{-g} d^{4} x\left\{\beta G_{\mu \nu} A^{\mu} A^{\nu}\right\}$ with respect to the metric $g^{\mu \nu}$, besides a bulk term $Z_{\mu \nu}$ in (2), gives the following boundary term

$$
\begin{aligned}
& \frac{\beta}{2 \kappa^{2}} \int d^{3} x \sqrt{-\gamma}\left\{\frac{1}{2} n_{\rho} \nabla^{\rho}\left(\delta g^{\alpha \beta}\right) A_{\alpha} A_{\beta}-A^{\mu} \nabla_{\mu}\left(\delta g^{\lambda \rho}\right) A_{\lambda} n_{\rho}\right. \\
& +\frac{1}{2} n_{\nu} A^{\nu} A^{\mu} \nabla_{\mu}\left(\delta g^{\lambda \rho}\right) g_{\lambda \rho}+\frac{1}{2} A^{2} \nabla_{\lambda}\left(\delta g^{\lambda \rho}\right) n_{\rho} \\
& \quad-\frac{1}{2} A^{2} n^{\alpha} \nabla_{\alpha}\left(\delta g^{\lambda \rho}\right) g_{\lambda \rho}+\delta g^{\lambda \rho} \nabla_{\rho}\left(A_{\lambda} A^{\nu}\right) n_{\nu} \\
& -\frac{1}{2} \delta g^{\alpha \beta} n^{\rho} \nabla_{\rho}\left(A_{\alpha} A_{\beta}\right)-\frac{1}{2} g_{\lambda \rho} \delta g^{\lambda \rho} n_{\mu} \nabla_{\nu}\left(A^{\mu} A^{\nu}\right) \\
& \left.-\frac{1}{2} n_{\lambda} \delta g^{\lambda \rho} \nabla_{\rho} A^{2}+\frac{1}{2} \delta g^{\lambda \rho} g_{\lambda \rho} n^{\alpha} \nabla_{\alpha} A^{2}\right\} .
\end{aligned}
$$

Note that there also exists the $\nabla^{\mu}\left(\delta g^{\alpha \beta}\right)$-like terms in (20), which are discontinuous across the boundary hypersurface. For obtaining the well-defined junction condition on the spacetime boundary, we involve the following boundary term

$$
\begin{aligned}
S_{\text {sur }}^{\beta}= & \frac{\beta}{2 \kappa^{2}} \int d^{3} x \sqrt{-\gamma}\left\{-A^{\nu}\left(\nabla_{\nu} A^{\rho}\right) n_{\rho}\right. \\
& \left.+n_{\nu} A^{\nu}\left(\nabla_{\rho} A^{\rho}\right)-A^{2} g^{\alpha \beta}\left(\nabla_{\beta} n_{\alpha}\right)\right\} .
\end{aligned}
$$

Thus the total boundary term is given as,

$$
S_{\mathrm{sur}}=S_{\mathrm{GH}}+S_{\mathrm{sur}}^{\beta}
$$

The variation of $S_{\text {sur }}^{\beta}$ with respect to $g^{\mu \nu}$ is

$$
\begin{aligned}
& \frac{\beta}{2 \kappa^{2}} \int d^{3} x \sqrt{-\gamma}\left\{-A^{\nu} n_{\rho} \delta \Gamma_{\nu \alpha}^{\rho} A^{\alpha}+n_{\nu} A^{\nu}\left(\delta \Gamma_{\rho \sigma}^{\rho} A^{\sigma}\right)\right. \\
& \quad-\frac{1}{2} A^{2}\left(\delta \Gamma_{\alpha \beta}^{\alpha} n^{\beta}\right)+\frac{1}{2} A^{2} g^{\alpha \beta}\left(\delta \Gamma_{\beta \alpha}^{\rho} n_{\rho}\right)+n_{\nu} A^{\nu} \delta g^{\rho \sigma}\left(\nabla_{\rho} A_{\sigma}\right) \\
& \quad+\frac{1}{2} \gamma_{\mu \nu} \delta g^{\mu \nu} A^{\alpha}\left(\nabla_{\alpha} A^{\rho}\right) n_{\rho}-\frac{1}{2} \gamma_{\alpha \beta} \delta g^{\alpha \beta} n_{\nu} A^{\nu}\left(\nabla_{\rho} A^{\rho}\right) \\
& \quad+\frac{1}{2} \gamma_{\mu \nu} \delta g^{\mu \nu} A^{2} g^{\alpha \beta}\left(\nabla_{\beta} n_{\alpha}\right)-\delta g^{\mu \nu} A_{\mu} A_{\nu} g^{\alpha \beta}\left(\nabla_{\beta} n_{\alpha}\right) \\
& \left.\quad-A^{2} \delta g^{\alpha \beta}\left(\nabla_{\beta} n_{\alpha}\right)-\delta g^{\mu \nu} A_{\mu}\left(\nabla_{\nu} A^{\rho}\right) n_{\rho}\right\}
\end{aligned}
$$

in which the $\delta \Gamma_{\mu \nu}^{\rho}$ is defined as

$$
\delta \Gamma_{\mu \nu}^{\rho}=-\frac{1}{2}\left(g_{\lambda \mu} \nabla_{\nu}\left(\delta g^{\lambda \rho}\right)+g_{\lambda \nu} \nabla_{\mu}\left(\delta g^{\lambda \rho}\right)-g_{\mu \alpha} g_{\nu \beta} \nabla^{\rho}\left(\delta g^{\alpha \beta}\right)\right)
$$

Adding the (19) with (20), (23) together, the quasilocal stress tensor on spacetime boundary could be given by the following junction condition

$$
\begin{aligned}
T_{a b}= & K_{a b}-K \gamma_{a b}+\beta\left(\frac{1}{2} \gamma_{a b} A^{2} K+\frac{1}{2} \gamma_{a b} n^{\rho} \nabla_{\rho} A^{2}\right. \\
& -A^{2} K_{a b}-\gamma_{a b} n_{\alpha} A^{\alpha} \nabla_{\beta} A^{\beta}+2 e_{a}^{\mu} e_{b}^{\nu} n_{\alpha} A^{\alpha} \nabla_{\mu} A_{\nu} \\
& \left.-e_{a}^{\mu} e_{b}^{\nu} n^{\rho} \nabla_{\rho} A_{\mu} A_{\nu}-e_{a}^{\mu} e_{b}^{\nu} A_{\mu} A_{\nu} K\right)
\end{aligned}
$$

where $K_{a b}=e_{a}^{\mu} e_{b}^{\nu} K_{\mu \nu} \quad$ and $\quad \gamma_{a b}=e_{a}^{\mu} e_{b}^{\nu} \gamma_{\mu \nu}=e_{a}^{\mu} e_{b}^{\nu} g_{\mu \nu}$. Meanwhile, the $T_{a b}$ is the stress tensor of the matter field which lives on the spacetime boundary, i.e., $T_{a b}=-\frac{2}{\sqrt{-\gamma}} \frac{\delta S_{\mathrm{CFT}}}{\delta \gamma^{a b}}$. Note that only the tangential components of the junction condition are nontrival, thus we multiply the vielbein $e_{a}^{\mu}$ in above equation. Actually, (24) could be viewed as the generalized Israel junction condition for this type of vector-tensor theory (1). The right-hand side (rhs) of (24) is derived from the geometry of bulk spacetime, while the $T_{a b}$ in 1.h.s corresponds to the stress tensor of the matter field living on the spacetime boundary.

\section{B. Surface counterterms and holographic energy}

As indicated in [66], the total energy of black hole system, i.e., the black hole mass, is defined as a conserved charge associated with a timelike killing vector,

$$
\begin{aligned}
M & =\int_{r \rightarrow \infty} d \Omega_{2}\left\{r^{2}(h(r))^{-\frac{1}{2}} T_{00}\right\} \\
& =-\left.\sqrt{5} \pi \frac{\left(Q_{2}^{2}-8\right)}{\Lambda} T_{00}\right|_{r \rightarrow \infty}
\end{aligned}
$$

where $T_{00}$ is the 00-components of stress tensor (24). However, the rhs of (24) typically diverges as $r \rightarrow \infty$. Specifically, substitute the solutions (10), (11), (12), (13) 
into (24) and then expand the results into power series, we could observe

$$
\left.T_{00}\right|_{r \rightarrow \infty}=c_{3} r^{3}+c_{1} r+c_{0}+O\left(\frac{1}{r}\right) \ldots
$$

in which the coefficients $c_{3}, c_{1}$ are constituted by $Q_{2}$ and $\Lambda$, while the $c_{0}$ consist of $Q, Q_{2}, m, \Lambda$.

From the viewpoint of gauge/gravity duality, the $T_{a b}$ is interpreted as the expectation value of the stress tensor in the side of boundary field theory [3,56-59]. And the UV (ultraviolet) divergences appear naturally in field theory as the energy scale increases, which is reflected by the divergences of geometry quantity given by the rhs of Eq. (24) as the boundary is taken to infinity. In the renormalization procedure of quantum field theory, all UV divergences could be removed by adding the counterterms to the bare Lagrangian. In the same spirit for gravity theory, a finite set of boundary terms could be constructed as the counterterms which only cancel the divergences on the boundary spacetime and do not change the equations of motion in the bulk spacetime.

But in our case, the surface counterterms will be more complicated than in [60], in which a standard expression of surface counterterms associated with a gravitational system in asymptotically AdS spacetime have been constructed.
First, besides the gravitational field and negative cosmology constant, there also exists the vector field which couples to the gravitational field in nonminimal ways. Thus, except the intrinsic geometry quantities on the spacetime boundary, the surface counterterms should also include the contributions from the vector field. Second, the black hole solution (10)-(11) is not asymptotically AdS. So, the standard results of counterterms for $\mathrm{AdS}_{4}$ given by [60] are not applicable directly in the current case, and some nonstandard results have to be developed.

Under $r \rightarrow \infty$ limit, for cancelling the divergences in stress tensor (24), we construct the following counterterms

$$
\begin{aligned}
S_{c t}= & \frac{-1}{\kappa^{2}} \int d^{3} x \sqrt{-\gamma}\left\{\frac{c_{0}}{\ell \sqrt{A^{\mu} A_{\mu}}}+\frac{c_{1} \ell \mathcal{R}}{\sqrt{A^{\mu} A_{\mu}}}+c_{m 1} n^{\mu} F_{\mu \nu} A^{\nu}\right. \\
& \left.+c_{m 2} \frac{n^{\mu} F_{\mu \nu} A^{\nu}}{A^{\alpha} A_{\alpha}}+c_{m 3} \ell \frac{\left(n^{\mu} F_{\mu \nu} A^{\nu}\right)^{2}}{\sqrt{A^{\alpha} A_{\alpha}}}\right\}
\end{aligned}
$$

where $\mathcal{R}$ is the Ricci scalar of the induced metric $\gamma_{a b}$ and $\ell$ is AdS radius related with cosmological constant through $\Lambda=-\frac{3}{\ell^{2}}$. Note that the $c_{0}, c_{1}, c_{m 1}, c_{m 2}, c_{m 3}$ are undetermined coefficients; they will be given later. With the inclusion of counterterms (27), the stress tensor will be rewritten as

$$
\begin{aligned}
T_{a b}^{\mathrm{eff}}= & T_{a b}^{0}+\frac{c_{0}}{\ell}\left(\frac{\gamma_{a b}}{\sqrt{A^{\mu} A_{\mu}}}+\frac{e_{a}^{\alpha} e_{b}^{\beta} A_{\alpha} A_{\beta}}{\sqrt{\left(A^{\mu} A_{\mu}\right)^{3}}}\right)+\ell c_{1}\left\{\frac{\gamma_{a b} \mathcal{R}}{\sqrt{A^{\mu} A_{\mu}}}-\frac{2 \mathcal{R}_{a b}}{\sqrt{A^{\mu} A_{\mu}}}+2\left(D_{a} D_{b} \frac{1}{\sqrt{A^{\mu} A_{\mu}}}-\gamma_{a b} D^{c} D_{c} \frac{1}{\sqrt{A^{\mu} A_{\mu}}}\right)+\frac{\mathcal{R} e_{a}^{\mu} e_{b}^{\nu} A_{\mu} A_{\nu}}{\sqrt{\left(A^{\mu} A_{\mu}\right)^{3}}}\right\} \\
& +c_{m_{1}}\left\{\gamma_{a b} n^{\mu} F_{\mu \nu} A^{\nu}-2 e_{(a}^{\nu} e_{b)^{\beta}}^{\beta} n^{\mu} F_{\mu \nu} A_{\beta}\right\}+c_{m_{2}}\left\{\gamma_{a b} \frac{n^{\mu} F_{\mu \nu} A^{\nu}}{A^{\rho} A_{\rho}}+2 e_{a}^{\beta} e_{b}^{\gamma} A_{\beta} A_{\gamma} \frac{n^{\mu} F_{\mu \nu} A^{\nu}}{\left(A^{\rho} A_{\rho}\right)^{2}}-2 e_{(a}^{\nu} e_{b)}^{\beta} \frac{n^{\mu} F_{\mu \nu} A_{\beta}}{A^{\rho} A_{\rho}}\right\} \\
& +c_{m_{3}} \ell\left\{e_{a}^{\rho} e_{b}^{\sigma} A_{\rho} A_{\sigma} \frac{\left(n^{\mu} F_{\mu \nu} A^{\nu}\right)^{2}}{\sqrt{\left(A^{\alpha} A_{\alpha}\right)^{3}}}-n^{\mu} F_{\mu \nu} A_{\beta} e_{(a}^{\nu} e_{b)}^{\beta} \frac{4 n^{\rho} F_{\rho \sigma} A^{\sigma}}{\sqrt{A^{\alpha} A_{\alpha}}}+\gamma_{a b} \frac{\left(n^{\mu} F_{\mu \nu} A^{\nu}\right)^{2}}{\sqrt{A^{\alpha} A_{\alpha}}}\right\}
\end{aligned}
$$

in which $D_{a}$ is the covariant differentiation with respect to induced metric $\gamma_{a b}$. Meanwhile, we use $T_{a b}^{0}$ to denote the bare stress tensor (24).

To remove the $O\left(r^{3}\right)$ and $O(r)$ divergences in the bare stress tensor $T_{a b}^{0}$, the undetermined coefficients are chosen as $c_{0}=\frac{32 \sqrt{5}}{5 \sqrt{3}}, c_{1}=\frac{44}{9 \sqrt{15}}, c_{m_{1}}=-\frac{7}{8}, c_{m_{2}}=-\frac{154}{15}$, $c_{m_{3}}=-\frac{5 \sqrt{5}}{32 \sqrt{3}}$. Substitute the above coefficients and (10), (11), (12), (13), (24) into the (28), we obtain

$$
\left.T_{00}^{\mathrm{eff}}\right|_{r \rightarrow \infty}=\frac{\left(5 m\left(Q_{2}^{2}-8\right)-3 Q Q_{2}\right) \Lambda}{2 \sqrt{5}\left(Q_{2}^{2}-8\right)}+O\left(\frac{1}{r}\right)+\ldots
$$

According to the above results and (25), the black hole mass is given as

$$
M=\frac{\pi}{2}\left(3 Q Q_{2}-5 m\left(Q_{2}^{2}-8\right)\right)
$$

\section{THERMODYNAMICS AND PHASE STRUCTURE}

\section{A. The first law of black hole thermodynamics}

From the metric ansatz (4), the Hawking temperature could be calculated as

$$
T=\left.\frac{\sqrt{h^{\prime} f^{\prime}}}{4 \pi}\right|_{r_{h}}=\frac{\left|4 \Lambda r_{h}^{2}+Q_{2}^{2}-8\right|}{4 \pi r_{h}\left(Q_{2}^{2}-8\right)} .
$$

As explained in Sec. II, we only consider $Q_{2}^{2}>8$ in this paper. For convenience in the following, we define $r_{h}^{\star}$ as the zero-point of expression (31). And it is easy to see, 


$$
T= \begin{cases}\frac{4 \Lambda r_{h}^{2}+Q_{2}^{2}-8}{4 \pi r_{h}\left(Q_{2}^{2}-8\right)}, & r_{h}<r_{h}^{\star} \\ 0, & r_{h}=r_{h}^{\star} \\ -\frac{4 \Lambda r_{h}^{2}+Q_{2}^{2}-8}{4 \pi r_{h}\left(Q_{2}^{2}-8\right)}, & r_{h}>r_{h}^{\star} .\end{cases}
$$

According to the area law, the entropy of black hole is

$$
S=\frac{A}{4 G}
$$

in which $A$ represents the area of event horizon. Note that the convention $\kappa=8 \pi G=1$ has been used in this paper, and the $S$ will be rewritten as

$$
S=2 \pi A=8 \pi^{2} r_{h}^{2}
$$

From the viewpoint of dynamics, the $Q$ is just an integration constant solved from the Einstein field equation and the extended Proca equation. In (12), (13), it seems that the value of $Q$ is free to choose and independent with other physical parameters like $Q_{2}, r_{h}, \Lambda$. However, for making the first law of black hole thermodynamics to hold,

$$
d M=T d S+\Phi d Q
$$

a constraint relation between $Q$ and $Q_{2}, r_{h}, \Lambda$ is implied,

$$
\begin{aligned}
Q= & \frac{8 r_{h}^{5} \Lambda^{2}}{3 Q_{2}\left(Q_{2}^{2}-8\right)}+\frac{5\left(Q_{2}^{2}-8\right) r_{h}}{6 Q_{2}}+\frac{20 r_{h}^{3} \Lambda}{9 Q_{2}} \\
& + \begin{cases}\frac{8\left(\frac{4}{3} \Lambda r_{h}^{3}+\left(Q_{2}^{2}-8\right) r_{h}\right)}{3 Q_{2}\left(Q_{2}^{2}-8\right)}, & r_{h} \leq r_{h}^{\star} \\
\frac{2 C_{0}\left(r_{h}^{\star}\right)}{3 Q_{2} \pi}-\frac{8\left(\frac{4}{3} \Lambda r_{h}^{3}+\left(Q_{2}^{2}-8\right) r_{h}\right)}{3 Q_{2}\left(Q_{2}^{2}-8\right)}, & r_{h} \geq r_{h}^{\star}\end{cases}
\end{aligned}
$$

in which the function $C_{0}\left(r_{h}^{\star}\right)$ has the following expression,

$$
C_{0}\left(r_{h}^{\star}\right)=\frac{8 \pi\left(\frac{4}{3} \Lambda r_{h}^{\star 3}+\left(Q_{2}^{2}-8\right) r_{h}^{\star}\right)}{\left(Q_{2}^{2}-8\right)}
$$

With thermodynamic quantities and constraint presented above, let us check that the (35) is established properly. Through the definition of horizon radius $h\left(r_{h}\right)=0$, a constraint relation between $m$ and $r_{h}, Q_{2}, \Lambda$ is deduced as follows

$$
m=\frac{r_{h}}{2}+\frac{4 \Lambda r_{h}^{3}}{3\left(Q_{2}^{2}-8\right)}+\frac{8 \Lambda^{2} r_{h}^{5}}{5\left(Q_{2}^{2}-8\right)^{2}}
$$

Substitute (36) and (37) into (30), we get

$$
M= \begin{cases}\frac{4 \pi\left(\frac{4}{3} \Lambda r_{h}^{3}+\left(Q_{2}^{2}-8\right) r_{h}\right)}{\left(Q_{2}^{2}-8\right)}, & r_{h} \leq r_{h}^{\star} \\ -\frac{4 \pi\left(\frac{4}{3} \Lambda r_{h}^{3}+\left(Q_{2}^{2}-8\right) r_{h}\right)}{\left(Q_{2}^{2}-8\right)}+C_{0}\left(r_{h}^{\star}\right), & r_{h} \geq r_{h}^{\star} .\end{cases}
$$

Combine (38) with (32), (34), it is easy to check

$$
\frac{\partial M}{\partial S}=\left(\frac{\partial M}{\partial r_{h}}\right) /\left(\frac{\partial S}{\partial r_{h}}\right)=T .
$$

Due to the breaking of $U(1)$ symmetry in Einstein-Proca theory, the electric charge is not the locally conserved charge anymore. And we could not obtain the electric charge directly from the Gauss law which does not hold in current situation. However, from the solution of electric potential (12), it is not difficult to observe that the $Q$ and $Q_{2}$ play the roles of charge and chemical potential respectively. In another way, from black hole mass (30), we could obtain the following relation directly,

$$
\frac{\partial M}{\partial Q}=\frac{3 \pi}{2} Q_{2}
$$

It also means that the $Q$ and $Q_{2}$ are a pair of conjugate variables in thermodynamics. Exactly, we define the chemical potential $\Phi$ as

$$
\Phi=\frac{3 \pi}{2} Q_{2}
$$

Note that the charge is not the conserved quantity in current situation, thus we consider the thermodynamics in the grand canonical ensemble.

As we known, for the Reissner-Nordström black hole solved from Einstein-Maxwell theory, $Q \leq M$, or the horizon does not exist. More generally, this extremality bound holds for most of charged black holes. From the black hole solution (10)-(13), it seems that the value of $Q$ is arbitrary and has no effect on the existence of horizon. However, after the consideration of thermodynamics, we find a constraint relation between $Q$ and other physical parameters, on which the first law of black hole thermodynamics could be reproduced correctly. Finally, by using (36) and (38) implied by thermodynamics, we can compare charge $Q$ and black hole mass $M$ at fixed horizon $r_{h}$, chemical potential $\Phi\left(Q_{2}\right)$ and cosmological constant $\Lambda$. As shown by Fig. 2, we observe that the value of $Q$ less than the value of $M$ indeed.

\section{B. Phase structures analysis}

According to the expression of the Hawking temperature (31), we plot the variation of temperature versus the horizon radius $r_{h}$ in Fig. 3. For a given temperature $T>0$, there exists two values of horizon $r_{h}$. We call them "small black hole" (SBH) and "large black hole" (LBH) phases, as shown by purple and red curves in Fig. 3 respectively.

Note that the SBH and LBH coexist at the same temperature, and we need to determine which phase is thermodynamically preferred. This goal could be achieved by comparing the Gibbs free energy of SBH and LBH; the 

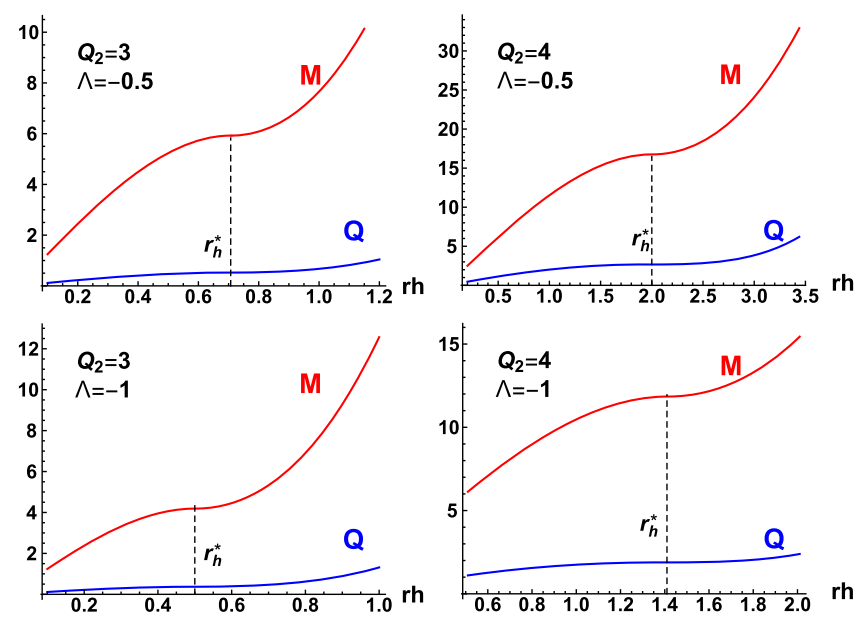

FIG. 2. Compare charge and black hole mass in some representative parameters.

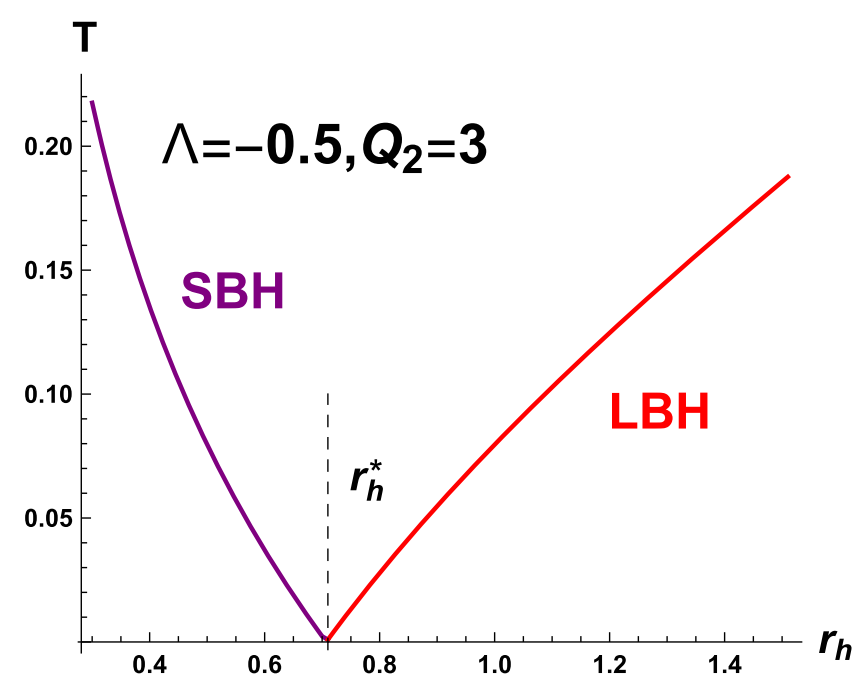

FIG. 3. The variation of temperature versus the horizon radius $r_{h}$ for the four-dimensional black hole solutions with spherical horizon, in representative parameters $\Lambda=-0.5$ and $Q_{2}=3$.

one which has lower free energy will be favored by the thermodynamical system. In the grand canonical ensemble, the Gibbs free energy is defined as

$$
G=M-T S-\Phi Q
$$

substitute (32), (34), (36), (38), (41) into (42), we have

$$
\begin{aligned}
G= & -\frac{5}{4}\left(Q_{2}^{2}-8\right) \pi r_{h}-\frac{10}{3} \pi r_{h}^{3} \Lambda-\frac{4 \pi r_{h}^{5} \Lambda^{2}}{Q_{2}^{2}-8} \\
& -2 \pi r_{h} \times \begin{cases}\frac{4 \Lambda r_{h}^{2}+Q_{2}^{2}-8}{\left(Q_{2}^{2}-8\right)}, & r_{h} \leq r_{h}^{\star} \\
-\frac{4 \Lambda r_{h}^{2}+Q_{2}^{2}-8}{\left(Q_{2}^{2}-8\right)}, & r_{h} \geq r_{h}^{\star}\end{cases}
\end{aligned}
$$

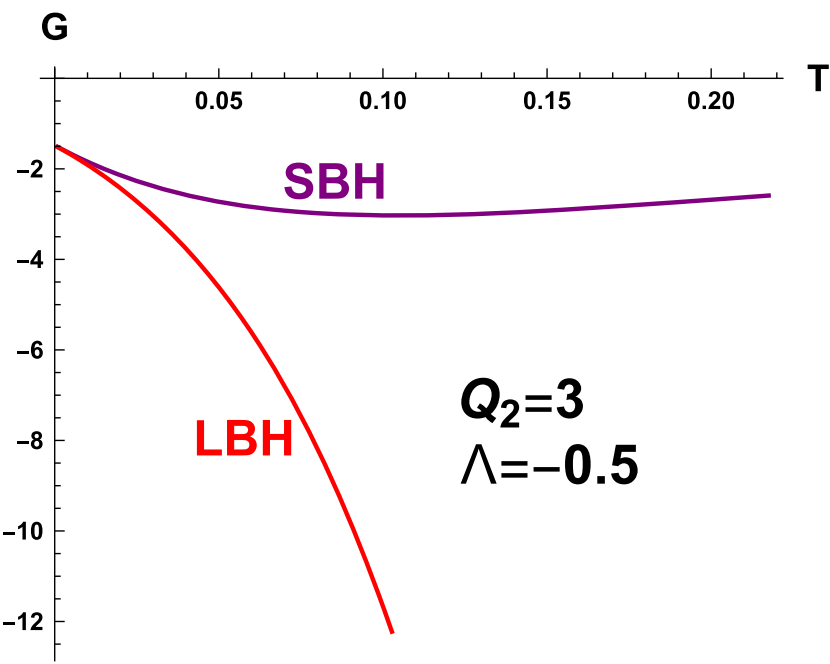

FIG. 4. The variation of Gibbs free energy $G$ versus the Hawking temperature $T$ for the four-dimensional black hole solutions with spherical horizon, in representative parameters $\Lambda=-0.5$ and $Q_{2}=3$.

In Fig. 4, we show that how Gibbs free energy $G$ varies with respect to the temperature $T$. It is easy to observe that the Gibbs free energy of LBH is lower than the one of SBH, thus the LBH is thermodynamically preferred. A similar conclusion could also be supported on comparing the specific heat of SBH and LBH. In the grand canonical ensemble, the specific heat is defined as

$$
C_{\Phi}=T\left(\frac{\partial S}{\partial T}\right)_{\Phi}=T\left(\frac{\partial S / \partial r_{h}}{\partial T / \partial r_{h}}\right)_{\Phi}
$$

substitute (31), (34) into (44) and expand it explicitly, we obtain

$$
C_{\Phi}=\frac{16 \pi^{2} r_{h}^{2}\left(8-Q_{2}^{2}-4 r_{h}^{2} \Lambda\right)}{Q_{2}^{2}-8-4 r_{h}^{2} \Lambda}
$$

From the $C_{\Phi}-T$ diagram, we easily see that the LBH phase has the positive specific heat, so it is thermodynamically stable. By contrast, the SBH is unstable.

Actually, the unstable SBH phase and stable LBH phase also imply a self-consistent result for thermodynamic quantities in extended phase space. In (38), we could identify that the branch in region $r_{h} \leq r_{h}^{\star}$ and $r_{h} \geq r_{h}^{\star}$ correspond to the SBH phase and $\mathrm{LBH}$ phase respectively. If we consider the black hole thermodynamics in extend phase space $[67,68]$, and define the black hole pressure $P$ as,

$$
P=-2 \pi \Lambda_{\text {eff }}=-\frac{4 \pi \Lambda}{Q_{2}^{2}-8} .
$$

Then, from (38), we can get 


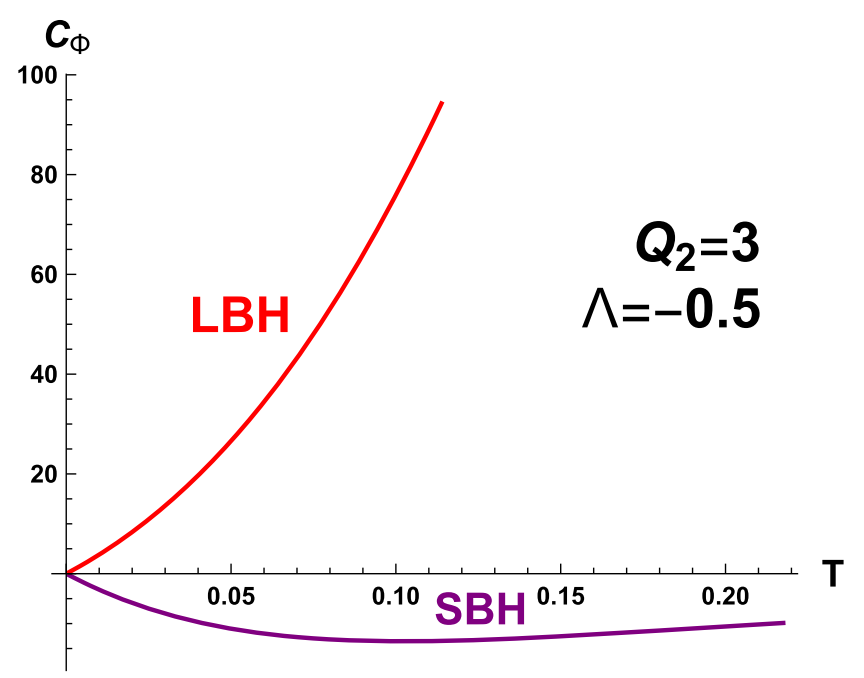

FIG. 5. The variation of specific heat $C_{\Phi}$ versus the Hawking temperature $T$ for the four-dimensional black hole solutions with spherical horizon, in representative parameters $\Lambda=-0.5$ and $Q_{2}=3$.

$$
\partial M / \partial P= \begin{cases}-\frac{4}{3} \pi r_{h}^{3}, & r_{h} \leq r_{h}^{\star} \\ \frac{4}{3} \pi r_{h}^{3}, & r_{h} \geq r_{h}^{\star}\end{cases}
$$

In $\mathrm{LBH}$ phase, the conjugate variables for pressure $P$ is exactly the black hole volume. But the result is unphysical in SBH phase. Thus we naturally expect that the SBH phase is not allowed by the thermodynamic system, in other words, this phase is unstable.

\section{CONCLUSIONS AND DISCUSSION}

For the type of nonminimally coupled vector-tensor theory given in [48], we computed and added the appropriate boundary terms (III A) to the bulk action (1) and derived the correspondingly well-defined junction condition (24) on the spacetime boundary. As interpreted by the gauge/gravity duality, the $T_{a b}$ in (24) represents the stress tensor of matter fields on the spacetime boundary. Meanwhile, this stress tensor is a divergent quantity. For removing the divergences in the stress tensor, surface counterterms need to be added to the total action. A standard expression of surface counterterms associated with a gravitational system in asymptotically AdS spacetime has been constructed by $[60,61]$, in which the surface counterterms consist of the cosmological constant and intrinsic geometry quantities of the boundary spacetime like the Ricci scalar and Ricci tensor for the boundary metric $\gamma_{a b}$. However, the surface counterterms in our case are more complicated than the results in [60,61], even though in four-dimensional spacetime. Our major difficulties come from the following two aspects. On the one hand, the gauge symmetry is broken due to the existence of nonminimal coupling between the vector field and Einstein tensor. On the other hand, the black hole solutions (10)(11) are not an asymptotic AdS geometry as shown by (15). Thus, unlike the expression of surface counterterms given by $[60,61]$, the mixture of vector field and intrinsic geometry quantities of boundary spacetime need to be considered in our case. Finally, after various attempts, we find the surface counterterms (27). Based on (27), we obtain the finite stress tensor (29) on boundary spacetime and calculate the corresponding conserved charge associated with a timelike killing vector, i.e., the black hole mass (30).

From (10)-(13), it is easy to see that the value of $Q$ is independent of other parameters and has no effect on the existence of a horizon. Thus, both $Q>M$ and $Q \leq M$ are allowed according to the expression (30). However, for most of charged black hole, there exists a general extremality bound $\frac{Q}{M} \leq 1$. Actually, after consideration of the first law of black hole thermodynamics, an implicit relation of $Q$ as the function of $Q_{2}, \Lambda$ and horizon $r_{h}$ are found in (36). Then, by using (36) and (38), we can compare the charge $Q$ with black hole mass $M$ directly at fixed $Q_{2}, \Lambda$ and $r_{h}$. As shown by Fig. 2, we indeed observe that the value of $Q$ less than the value of $M$. Besides, after the analysis of phase structure in grand canonical ensemble, we observe the unstabel $\mathrm{SBH}$ phase and stable LBH as displayed by Fig. 3-Fig. 5. In fact, as we show in (47), the unstable SBH phase and stable LBH phase also imply a self-consistent result for well-defined pressure and volume in extended phase space.

As future research, we suggest the following extended topics. In [37], inflation is driven by nonminimally coupled massive vector fields. It would be interesting consider cosmic inflation by using the vector-tensor theory (1). Besides, it is also worthwhile to develop the supersymmetric extension of (1) and investigate the relevant phenomenology in black hole physics and cosmology.

\section{ACKNOWLEDGMENTS}

We would like to thank C. Herdeiro and E. Radu, $\mathrm{Ru}$-yong Li for fruitful and useful discussions. This work is supported by The Center for Research and Development in Mathematics and Applications (CIDMA) through the Portuguese Foundation for Science and Technology (FCT_Fundao para a Cincia e a Tecnologia), references UIDB/04106/2020 and UIDP/04106/2020. Besides, A.-c.L is also partly supported by NSFC Grant No. 11875082. 
[1] J. M. Maldacena, The Large N limit of superconformal field theories and supergravity, Int. J. Theor. Phys. 38, 1113 (1999); Adv. Theor. Math. Phys. 2, 231 (1998).

[2] S. S. Gubser, I. R. Klebanov, and A. M. Polyakov, Gauge theory correlators from noncritical string theory, Phys. Lett. B 428, 105 (1998).

[3] E. Witten, Anti-de Sitter space and holography, Adv. Theor. Math. Phys. 2, 253 (1998).

[4] U. Gursoy, E. Kiritsis, L. Mazzanti, and F. Nitti, Deconfinement and Gluon Plasma Dynamics in Improved Holographic QCD, Phys. Rev. Lett. 101, 181601 (2008).

[5] U. Gursoy, E. Kiritsis, L. Mazzanti, and F. Nitti, Holography and thermodynamics of 5D dilaton-gravity, J. High Energy Phys. 05 (2009) 033.

[6] S. S. Gubser, A. Nellore, S. S. Pufu, and F. D. Rocha, Thermodynamics and Bulk Viscosity of Approximate Black Hole Duals to Finite Temperature Quantum Chromodynamics, Phys. Rev. Lett. 101, 131601 (2008).

[7] S. A. Hartnoll, C. P. Herzog, and G. T. Horowitz, Building a Holographic Superconductor, Phys. Rev. Lett. 101, 031601 (2008).

[8] S. A. Hartnoll, C. P. Herzog, and G. T. Horowitz, Holographic superconductors, J. High Energy Phys. 12 (2008) 015 .

[9] S. A. Hartnoll and P. Kovtun, Hall conductivity from dyonic black holes, Phys. Rev. D 76, 066001 (2007).

[10] S. A. Hartnoll and C. P. Herzog, Ohm's Law at strong coupling: $S$ duality and the cyclotron resonance, Phys. Rev. D 76, 106012 (2007).

[11] D. Astefanesei, R. B. Mann, and R. Rojas, Hairy black hole chemistry, J. High Energy Phys. 11 (2019) 043.

[12] R. A. Hennigar, R. B. Mann, and E. Tjoa, Superfluid Black Holes, Phys. Rev. Lett. 118, 021301 (2017).

[13] Y. M. Xu, H. M. Wang, Y. X. Liu, and S. W. Wei, Photon sphere and reentrant phase transition of charged BornInfeld-AdS black holes, Phys. Rev. D 100, 104044 (2019).

[14] S. Mahapatra, S. Priyadarshinee, G. N. Reddy, and B. Shukla, Exact topological charged hairy black holes in AdS Space in D-dimensions, Phys. Rev. D 102, 024042 (2020).

[15] R. G. Cai, J. Y. Ji, and K. S. Soh, Hairs on the cosmological horizons, Phys. Rev. D 58, 024002 (1998).

[16] C. Martinez, R. Troncoso, and J. Zanelli, De Sitter black hole with a conformally coupled scalar field in fourdimensions, Phys. Rev. D 67, 024008 (2003).

[17] K. G. Zloshchastiev, On Co-Existence of Black Holes and Scalar Field, Phys. Rev. Lett. 94, 121101 (2005).

[18] E. Winstanley, Dressing a black hole with non-minimally coupled scalar field hair, Classical Quantum Gravity 22, 2233 (2005).

[19] Y. Brihaye and T. Delsate, Skyrmion and Skyrme-black holes in de Sitter spacetime, Mod. Phys. Lett. A 21, 2043 (2006).

[20] Y. Brihaye, B. Hartmann, E. Radu, and C. Stelea, Cosmological monopoles and non-abelian black holes, Nucl. Phys. B763, 115 (2007).

[21] C. A. R. Herdeiro and E. Radu, Kerr Black Holes with Scalar Hair, Phys. Rev. Lett. 112, 221101 (2014).

[22] C. A. R. Herdeiro and E. Radu, A new spin on black hole hair, Int. J. Mod. Phys. D 23, 1442014 (2014).
[23] C. L. Benone, L. C. B. Crispino, C. Herdeiro, and E. Radu, Kerr-Newman scalar clouds, Phys. Rev. D 90, 104024 (2014).

[24] Y. Brihaye, C. Herdeiro, and E. Radu, MyersPerry black holes with scalar hair and a mass gap, Phys. Lett. B 739, 1 (2014).

[25] C. Herdeiro, E. Radu, and H. Rnarsson, Kerr black holes with Proca hair, Classical Quantum Gravity 33, 154001 (2016).

[26] G. Antoniou, A. Bakopoulos, and P. Kanti, Evasion of No-Hair Theorems and Novel Black-Hole Solutions in Gauss-Bonnet Theories, Phys. Rev. Lett. 120, 131102 (2018).

[27] G. Antoniou, A. Bakopoulos, and P. Kanti, Black-hole solutions with scalar hair in Einstein-Scalar-Gauss-Bonnet theories, Phys. Rev. D 97, 084037 (2018).

[28] H. O. Silva, J. Sakstein, L. Gualtieri, T. P. Sotiriou, and E. Berti, Spontaneous Scalarization of Black Holes and Compact Stars from a Gauss-Bonnet Coupling, Phys. Rev. Lett. 120, 131104 (2018).

[29] D. D. Doneva and S. S. Yazadjiev, New Gauss-Bonnet Black Holes with Curvature-Induced Scalarization in Extended Scalar-Tensor Theories, Phys. Rev. Lett. 120, 131103 (2018).

[30] D. D. Doneva, S. Kiorpelidi, P. G. Nedkova, E. Papantonopoulos, and S.S. Yazadjiev, Charged GaussBonnet black holes with curvature induced scalarization in the extended scalar-tensor theories, Phys. Rev. D 98, 104056 (2018).

[31] M. Minamitsuji and T. Ikeda, Scalarized black holes in the presence of the coupling to Gauss-Bonnet gravity, Phys. Rev. D 99, 044017 (2019).

[32] H. O. Silva, C. F. B. Macedo, T. P. Sotiriou, L. Gualtieri, J. Sakstein, and E. Berti, Stability of scalarized black hole solutions in scalar-Gauss-Bonnet gravity, Phys. Rev. D 99, 064011 (2019).

[33] C. F. B. Macedo, J. Sakstein, E. Berti, L. Gualtieri, H. O. Silva, and T. P. Sotiriou, Self-interactions and spontaneous black hole scalarization, Phys. Rev. D 99, 104041 (2019).

[34] P. V. P. Cunha, C. A. R. Herdeiro, and E. Radu, Spontaneously Scalarized Kerr Black Holes in Extended ScalarTensorGauss-Bonnet Gravity, Phys. Rev. Lett. 123, 011101 (2019).

[35] C. A. R. Herdeiro and E. Radu, Black hole scalarization from the breakdown of scale invariance, Phys. Rev. D 99, 084039 (2019).

[36] C. A. R. Herdeiro, E. Radu, N. Sanchis-Gual, and J. A. Font, Spontaneous Scalarization of Charged Black Holes, Phys. Rev. Lett. 121, 101102 (2018).

[37] A. Golovnev, V. Mukhanov, and V. Vanchurin, Vector inflation, J. Cosmol. Astropart. Phys. 06 (2008) 009.

[38] J. Beltrn Jimnez and A. L. Maroto, Dark energy, nonminimal couplings and the origin of cosmic magnetic fields, J. Cosmol. Astropart. Phys. 12 (2010) 025.

[39] G. Esposito-Farese, C. Pitrou, and J. P. Uzan, Vector theories in cosmology, Phys. Rev. D 81, 063519 (2010).

[40] G. Tasinato, Cosmic acceleration from Abelian symmetry breaking, J. High Energy Phys. 04 (2014) 067. 
[41] G. Tasinato, A small cosmological constant from Abelian symmetry breaking, Classical Quantum Gravity 31, 225004 (2014).

[42] R. B. Mann and R. McNees, Holographic renormalization for asymptotically Lifshitz spacetimes, J. High Energy Phys. 10 (2011) 129.

[43] C. Herdeiro, M. O. P. Sampaio, and M. Wang, Hawking radiation for a Proca field in $D$ dimensions, Phys. Rev. D 85, 024005 (2012).

[44] A. Ovgn and K. Jusufi, The effect of the GUP on massive vector and scalar particles tunneling from a warped DGP gravity black hole, Eur. Phys. J. Plus 132, 298 (2017).

[45] A. De Felice, L. Heisenberg, R. Kase, S. Mukohyama, S. Tsujikawa, and Y.1. Zhang, Cosmology in generalized Proca theories, J. Cosmol. Astropart. Phys. 06 (2016) 048.

[46] M. Zhou, C. Bambi, C. A. R. Herdeiro, and E. Radu, Iron $\mathrm{K} \alpha$ line of Kerr black holes with Proca hair, Phys. Rev. D 95, 104035 (2017).

[47] G. M. Deng, J. Fan, X. Li, and Y. C. Huang, Thermodynamics and phase transition of charged AdS black holes with a global monopole, Int. J. Mod. Phys. A 33, 1850022 (2018).

[48] J. Chagoya, G. Niz, and G. Tasinato, Black holes and Abelian symmetry breaking, Classical Quantum Gravity 33, 175007 (2016).

[49] B. M. Gripaios, Modified gravity via spontaneous symmetry breaking, J. High Energy Phys. 10 (2004) 069.

[50] L. Heisenberg, Generalization of the Proca action, J. Cosmol. Astropart. Phys. 05 (2014) 015.

[51] C. Herdeiro and E. Radu, Construction and physical properties of Kerr black holes with scalar hair, Classical Quantum Gravity 32, 144001 (2015).

[52] J. F. Delgado, C. A. Herdeiro, and E. Radu, Violations of the Kerr and Reissner-Nordstrm bounds: Horizon versus asymptotic quantities, Phys. Rev. D 94, 024006 (2016).

[53] Z. Y. Fan, Black holes in vector-tensor theories and their thermodynamics, Eur. Phys. J. C 78, 65 (2018).

[54] H. S. Liu, H. Lü, and C. N. Pope, Thermodynamics of Einstein-Proca AdS black holes, J. High Energy Phys. 06 (2014) 109.
[55] G. W. Gibbons and S. W. Hawking, Action integrals and partition functions in quantum gravity, Phys. Rev. D 15, 2752 (1977).

[56] M. Henningson and K. Skenderis, The holographic Weyl anomaly, J. High Energy Phys. 07 (1998) 023.

[57] S. Hyun, W. T. Kim, and J. Lee, Statistical entropy and AdS/CFT correspondence in BTZ black holes, Phys. Rev. D 59, 084020 (1999).

[58] G. Chalmers and K. Schalm, Holographic normal ordering and multiparticle states in the AdS/CFT correspondence, Phys. Rev. D 61, 046001 (2000).

[59] S. Nojiri and S. D. Odintsov, Conformal anomaly for dilaton coupled theories from AdS/CFT correspondence, Phys. Lett. B 444, 92 (1998).

[60] V. Balasubramanian and P. Kraus, A stress tensor for antide Sitter gravity, Commun. Math. Phys. 208, 413 (1999).

[61] R. Emparan, C. V. Johnson, and R. C. Myers, Surface terms as counterterms in the AdS/CFT correspondence, Phys. Rev. D 60, 104001 (1999).

[62] S. de Haro, S. N. Solodukhin, and K. Skenderis, Holographic reconstruction of space-time and renormalization in the AdS/CFT correspondence, Commun. Math. Phys. 217, 595 (2001).

[63] W. Israel, Singular hypersurfaces and thin shells in general relativity, Nuovo Cim. B 44S10, 1 (1966); 44, 1 (1966); Erratum, Nuovo Cim. B 48, 463 (1967).

[64] H. A. Chamblin and H. S. Reall, Dynamic dilatonic domain walls, Nucl. Phys. B562 (1999) 133

[65] A. C. Li, W. L. Xu, and D. F. Zeng, Linear stability analysis of evolving thin shell wormholes, J. Cosmol. Astropart. Phys. 03 (2019) 016.

[66] J. D. Brown and J. W. York, Jr., Quasilocal energy and conserved charges derived from the gravitational action, Phys. Rev. D 47, 1407 (1993).

[67] D. Kastor, S. Ray, and J. Traschen, Enthalpy and the mechanics of AdS black holes, Classical Quantum Gravity 26, 195011 (2009).

[68] D. Kubiznak and R. B. Mann, P-V criticality of charged AdS black holes, J. High Energy Phys. 07 (2012) 033. 